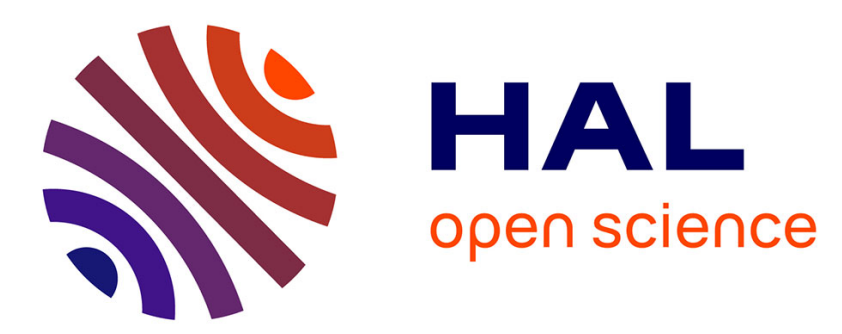

\title{
Control Surface Geometry Surrogate-Based Optimization for Spin-Stabilized Projectile Course Correction
}

\author{
Guillaume Arnoult, Mickael Zeidler, Éric Garnier
}

\section{To cite this version:}

Guillaume Arnoult, Mickael Zeidler, Éric Garnier. Control Surface Geometry Surrogate-Based Optimization for Spin-Stabilized Projectile Course Correction. AIAA Journal, 2019, 58 (2), pp.550-560. 10.2514/1.J058323 . hal-02906140

\section{HAL Id: hal-02906140 \\ https://hal.science/hal-02906140}

Submitted on 24 Jul 2020

HAL is a multi-disciplinary open access archive for the deposit and dissemination of scientific research documents, whether they are published or not. The documents may come from teaching and research institutions in France or abroad, or from public or private research centers.
L'archive ouverte pluridisciplinaire HAL, est destinée au dépôt et à la diffusion de documents scientifiques de niveau recherche, publiés ou non, émanant des établissements d'enseignement et de recherche français ou étrangers, des laboratoires publics ou privés. 


\title{
Control Surface Geometry Surrogate-Based Optimization for Spin-Stabilized Projectile Course Correction
}

\author{
Guillaume Arnoult* and Mickael Zeidler ${ }^{\dagger}$ \\ Nexter Munitions, 18023 Bourges, France \\ Eric Garnier \\ ONERA, 92190 Meudon, France
}

In the present paper a surrogate-based methodology is developed and applied to the design of a control surface placed on an artillery projectile. An artificial neural network is used to estimate the aerodynamic contribution of the control device from numerical simulations. These estimations are coupled to a flight mechanics code to evaluate the trajectories of controlled projectiles. From these 6/7 degrees of freedom computations, the trajectories modifications are modeled using kriging. A correction device should minimize the distance to a $3 \sigma$ ellipse (with $\sigma$ the projectile standard deviation of the projectile dispersion) around the mean impact point while ensuring problem specific constraints fulfillment. The kriging databases are sequentially enriched with the impact point position of the projectile configurations maximizing the Expected Improvement criterion until a convergence state, assessed by Leave One Out Cross Validation, is reached. CFD based evaluations of the optimum configuration coefficients provide a refinement of the neural network databases in the relevant area of the design space. A course correction corresponding to a decrease of 2 standard deviation in the lateral direction and an increase of $\mathbf{2}$ standard deviation in range is achieved respectively by reducing the pitching moment of the projectile and increasing its lift-to-drag ratio.

\section{Nomenclature}

$\alpha$

*Ph.D. student, Département Métier Agression Aérobalistique, guillaume.arnoult@onera.fr

${ }^{\dagger}$ Research Engineer, Département Agression Létalité Aérobalistique, m.zeidler@nexter-group.fr

${ }^{\ddagger}$ Research Scientist, Department of Aerodynamics, Aeroelasticity and Acoustics, eric.garnier@onera.fr 


\begin{tabular}{|c|c|c|}
\hline$C_{N}$ & $=$ & Normal force coefficient \\
\hline$C_{m}$ & $=$ & Pitching moment coefficient \\
\hline$\Delta C_{A}$ & $=$ & Spoiler contribution to the axial force coefficient \\
\hline$\Delta C_{N}$ & $=$ & Spoiler contribution to the normal force coefficient \\
\hline$\Delta C_{m}$ & $=$ & Spoiler contribution to the pitching moment coefficient \\
\hline$C_{N_{\alpha}}$ & $=$ & Gradient of normal force coefficient, $\mathrm{rad}^{-1}$ \\
\hline$C_{m_{\alpha}}$ & $=$ & Gradient of pitching moment coefficient, $\mathrm{rad}^{-1}$ \\
\hline$D$ & $=$ & Reference diameter, $m$ \\
\hline$X_{S} / D$ & $=$ & Spoiler longitudinal position w.r.t. the diameter \\
\hline$H_{s} / D$ & $=$ & Spoiler height w.r.t. the diameter \\
\hline$\theta$ & $=$ & Spoiler span angle, deg \\
\hline$\phi$ & $=$ & Spoiler roll position, deg \\
\hline$F_{s p}$ & $=$ & Additional force produced by the spoiler, $N$ \\
\hline$X$ & $=$ & Downrange, $m$ \\
\hline Y & $=$ & Altitude, $m$ \\
\hline$Z$ & $=$ & Crossrange, $m$ \\
\hline$X_{U P}$ & $=$ & Uncontrolled projectile downrange, $m$ \\
\hline$Y_{U P}$ & $=$ & Uncontrolled projectile altitude, $m$ \\
\hline$Z_{U P}$ & $=$ & Uncontrolled projectile crossrange, $m$ \\
\hline$X_{C P}$ & $=$ & Controlled projectile downrange, $m$ \\
\hline$Y_{C P}$ & $=$ & Controlled projectile altitude, $m$ \\
\hline$Z_{C P}$ & $=$ & Controlled projectile crossrange, $m$ \\
\hline$X_{3 \sigma}$ & $=$ & Nearest $3 \sigma$ ellipse point downrange, $m$ \\
\hline$Z_{3 \sigma}$ & $=$ & Nearest $3 \sigma$ ellipse point crossrange, $m$ \\
\hline$P_{U P}$ & $=$ & Uncontrolled projectile impact point position \\
\hline$P_{C P}$ & $=$ & Controlled projectile impact point position \\
\hline$P_{3 \sigma}$ & $=$ & Nearest $3 \sigma$ ellipse point position \\
\hline$\sigma_{X}$ & $=$ & Downrange error standard deviation of the projectile dispersion, $m$ \\
\hline$\sigma_{Z}$ & $=$ & Crossrange error standard deviation of the projectile dispersion, $m$ \\
\hline$p$ & $=$ & Problem dimensions \\
\hline$N$ & $=$ & Number of samples \\
\hline$N_{\text {init }}$ & $=$ & Initial sampling database \\
\hline
\end{tabular}




\begin{tabular}{|c|c|c|}
\hline$x$ & $=$ & Unsampled point location \\
\hline$x_{i}$ & $=$ & $i^{t h}$ sampled point of the database \\
\hline$y(x)$ & $=$ & Accurate evaluation of a function at location $x$ \\
\hline$y_{\min }$ & $=$ & Best evaluation of a function \\
\hline$\hat{y}(x)$ & $=$ & Estimation of a function value at location $x$ \\
\hline$R^{2}$ & $=$ & Determination coefficient \\
\hline$\mu$ & $=$ & Mean value of the dataset \\
\hline$Z$ & $=$ & Gaussian correlation process \\
\hline$\sigma$ & $=$ & Model variance \\
\hline$r$ & $=$ & Correlation vector \\
\hline$R$ & $=$ & Correlation matrix \\
\hline$s$ & $=$ & Root mean square estimation error \\
\hline$E I$ & $=$ & Expected Improvement \\
\hline$\Phi$ & $=$ & Cumulative distribution function \\
\hline$\phi$ & $=$ & Standard normal density function \\
\hline$g$ & $=$ & Expression of the constraint function \\
\hline$g_{\min }$ & $=$ & Limit of the constraint defining feasible designs \\
\hline$\hat{g}(x)$ & $=$ & Estimation of the constraint function at location $x$ \\
\hline$G(x)-g_{\min }$ & $=$ & Measure of the feasibility of design $x$ \\
\hline
\end{tabular}

\section{Introduction}

RTILLERY projectiles are subjects to external perturbations of different nature (for instance wind gusts, variation
of initial velocity etc) and therefore not deterministic. This results in the dispersion of the impact locations characterizing the ammunition precision error. The ability of a control surface to correct the projectile course is studied. The design of a spoiler deployed during a spinning projectile flight is studied with the aim of controlling the precision error. The control of the projectile course leads to an increased accuracy of the ammunition impact point position.

The evaluation of course correction relies on the computation of the projectile trajectory which depends on the estimation of the aerodynamic coefficients during the process. Dietrich [1] studied the coupling of a flight mechanic code with different levels of fidelity for the aerodynamic coefficients computation. Several approaches were investigated, from empirical and semi-empirical modeling (Spinner [2]) which allows the determination of a trajectory in about 10 minutes to RANS-based Computational Fluid Dynamics (CFD) which requires about one month physical-time of intensive CFD calculations for the same trajectory. However, empirical models tend to under-estimate the axial force 
coefficient resulting in an overestimation of the projectile range. The error can reach 3 to $10 \%$ compared to the real trajectory while the CFD computations lead to more precise evaluations of the coefficients producing an error of about $2 \%$ for the range and lateral deviation [1].

To overcome the RANS-based CFD computational time and the empirical models lack of accuracy, an alternative consists in modeling the variations of the aerodynamic coefficients using surrogate modeling, also called response surface methods. The flight mechanics code inputs are directly interpolated in the response surface constructed from a limited number of accurate but expensive calculations, reducing the computational cost of the complete aerodynamic model.

The interest for surrogate models in this paper is twofold. As a first step, aerodynamic coefficients are simply estimated from CFD-based response surfaces. Paiva et al. [3], Peter et al. [4] and Rajkumar and Burdina [5] compared different surrogate models ability in reproducing various function variations. From these studies, neural networks and kriging seems the most able to model the evolution of a function from a reduced sampling of CFD evaluations. Subsequently, several studies rely on surrogate models to identify optimum designs. Of specific interest in the present study, kriging appears promising in the case of an optimization study. The maximization of the expected improvement, calculated from the kriging model features has been successfully applied several times for the identification of design maximizing lift coefficient (Meunier [6] and Kanazaki et al. [7]) and for the lift-to-drag ratio of an airfoil (Jeong et al. [8] and Forrester et al. [9]). In the specific field of projectile optimization, surrogate models have been mostly used for missiles and projectiles range extension (Schönning et al. [10] and Won et al. [11]). Fowler and Rogers [12] mixed kriging and neural network with the aim of extending a projectile range while ensuring its stability during the flight.

Based on previous studies, the present work takes advantages of the combination of different surrogates models to solve an optimization problem. The originality of this study comes from the coupling between CFD evaluations and flight mechanics in a shape optimization framework. Fast aerodynamic coefficients evaluations are available thanks to a multi-layer perceptron (MLP) and its combination with kriging leads to the determination of controlled projectile configurations producing a course correction in both cross- and downrange with a sole device. A sequential enrichment of both surrogate models is developed at different steps of the optimization process to help the optimizer converging to an optimum. The refinement of the different response surfaces used during this study has proven to be necessary to accurately compute the trajectories and hence to determine an optimum device shape for the spin-stabilized projectile course correction.

The present paper is organized as follow : the selected device geometry is presented in section $\Pi$ Kriging and neural network methodologies are described briefly in section III] Aerodynamics of the controlled projectile are introduced in the section IV The optimization of the device design is presented in section $\mathrm{V}$ and a flight mechanics study is achieved in section VI to highlight the projectile behavior modifications. 


\section{Maneuver concept}

The choice of deploying a spoiler during the projectile flight has been particularly motivated by the work of Patel et al. [13] and Simon [14]. In their work, Patel et al. demonstrated the effectiveness of micro-spoiler in the capability of modifying the behavior of a projectile for an angle of attack ranging from 0 to $18^{\circ}$, which is the effective range of the present uncontrolled projectile. Simon demonstrated the ability of a spoiler to generate additional forces and moments from subsonic to supersonic regimes by using RANS and ZDES simulations.

The geometrical configuration of the spoiler is presented in fig. 1. Four parameters were optimized, the longitudinal position from the nose $X_{s} / D$, the height $H_{s} / D$, the span angle $\theta_{s}$ and the roll position $\phi_{s}$ defined w.r.t. the vertical axis in fig. 1p. The spoiler is supposed mounted on a free-rolling support and the latter parameter would be controlled by an internal motor providing the opposite spin-rate of the projectile. The angular position of the spoiler, deduced from the required 2-D course correction, would be fixed in the earth referential during the flight while the projectile body would be spinning. A unique deployment instant is chosen for the spoiler. From this time until the impact, the device is assumed deployed and maintained in position by the internal motor. The geometrical parameters ranges are given in table 1

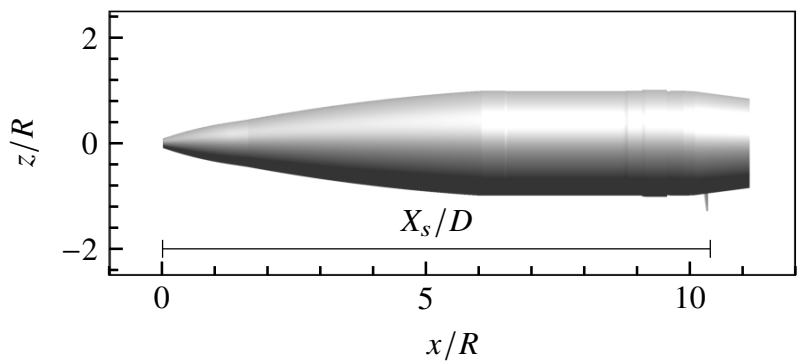

(a) Sideview projectile geometry

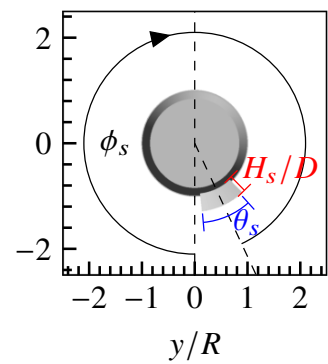

(b) Rear view of the projectile geometry

\section{Fig. 1 Visualization of the spoiler geometry}

The control of the angular position enables choosing toward which direction the trajectory is altered. A 2-D course correction combination of cross- down-range modifications ability is thus demonstrated by varying the roll position at which a given spoiler geometry is deployed in fig. 2 As illustrated by the arrows $F_{s}$, the normal force induced by the spoiler is continuously diametrically opposed to the spoiler angular position. During the projectile flight and until the spoiler deployment, information about the trajectory perturbation would be gathered using an external radar system. From the tracking system, the precision error at the impact is estimated and the course correction required is deduced.

The optimization problem consists in determining a design which produces a 2-D course correction reducing the precision error at the impact of the projectile. This error is statistically defined by STANAG 4635 [15] as the deviation of the real impact points from the mean impact point. It is determined as the quadratic sum of different errors occurring 

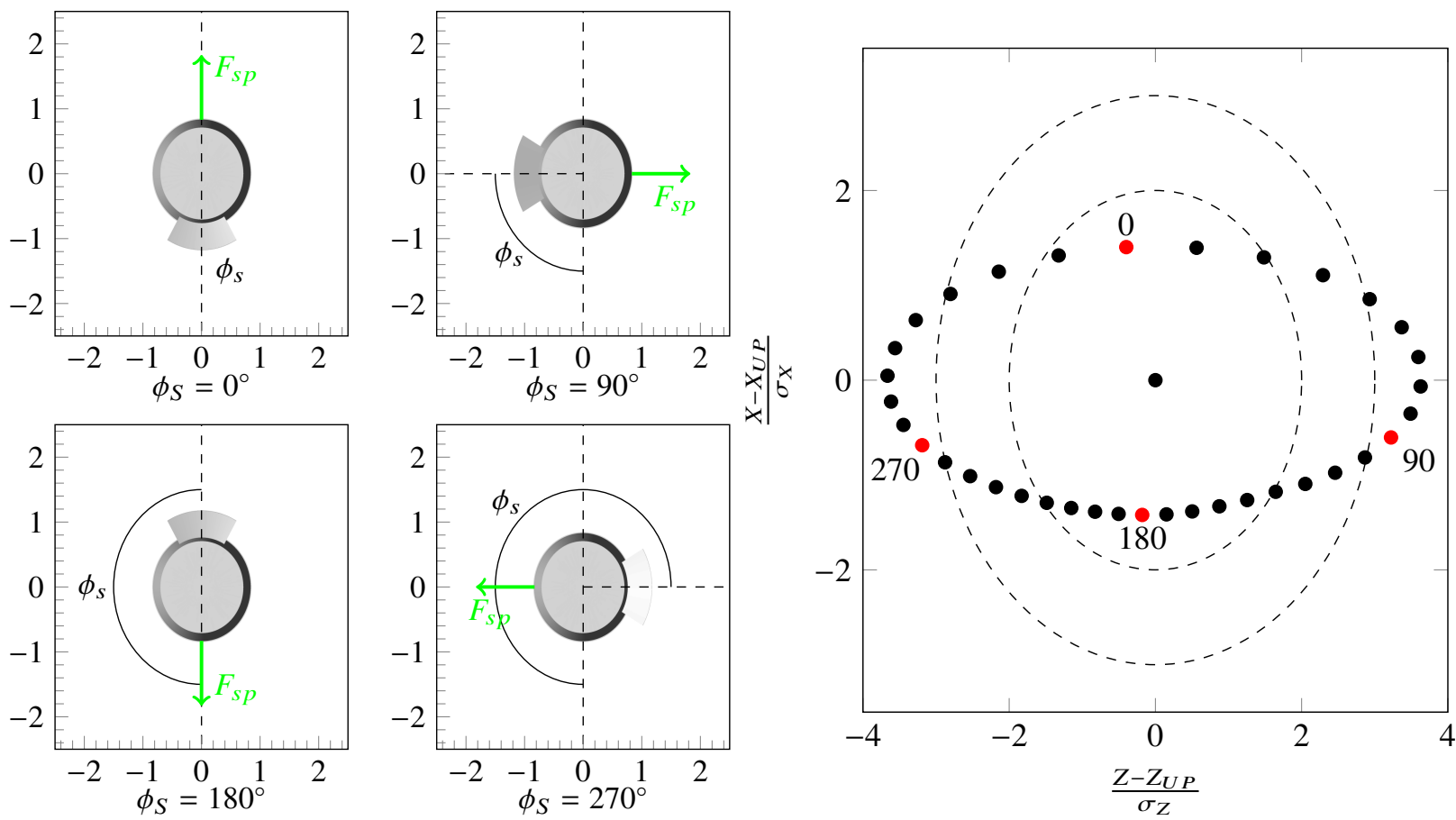

(a) Particular spoiler roll positions $\phi_{s}$

(b) Impact points for different roll positions $\phi_{S}$

Fig. 2 Spoiler roll position $\phi_{s}$ influence on the trajectory modification, the red dots are the impacts points associated to the roll positions presented in fig. $2 \mathrm{a}$

during the projectile flight (meteorological effects, variations in initial velocity, etc). The error budgets are empirically deduced from field firing conducted by DGA (French General Armament Direction) of the uncontrolled projectile. The precision error is assumed to follow a Gaussian distribution which is represented in fig. 2 by the dashed ellipses characterized by the down- and cross-range standard deviations.

\begin{tabular}{|c|c|c|}
\hline & \multicolumn{2}{|c|}{ Parameter range } \\
\cline { 2 - 3 } & $\min$ & $\max$ \\
\hline Longitudinal position $X_{S} / D$ & 4.85 & 5.5 \\
\hline Height of the spoiler $H_{S} / D$ & 0.05 & 0.25 \\
\hline Span of the spoiler $\theta_{s}\left(^{\circ}\right)$ & 15 & 60 \\
\hline Roll position $\phi_{s}\left(^{\circ}\right)$ & 0 & 360 \\
\hline
\end{tabular}

Table 1 Optimization parameters range

The additional forces and moment induced by the spoiler deployment enable the control of the projectile trajectory. In contrast with other control devices, the spoiler would remain deployed with its angular position fixed by the motor. Wey et al. [16] have highlighted that its deployment induces a transitional phase during which the forces and moments exerting on the projectile are not at an equilibrium. This transitional phase in not modeled in the present study. Since the spoiler position is time-invariant, the projectile reaches an equilibrium after a short period of time. This equilibrium 
position is located in the projectile resistance plane, which is also the spoiler symmetry plane.

\section{Surrogate models}

As estimated from the work of Dietrich [1], the accurate computation of aerodynamic coefficients along the trajectory is incompatible with the optimization framework presented in this study. Surrogate models are used to model the spoiler contribution to the aerodynamic coefficients and the trajectory modifications induced by the different parameters combinations. A short description of the models used in this study is presented here.

\section{A. Initial sampling}

In this study, Latin Hypercube Sampling LHS [17, 18] is used for its simplicity and its good overall performances. According to Jones et al. [19], a good initial sampling size counts 10 samples in every dimension which is usually not reachable on high-dimensional problems. From a practical point of view, only the spreading of $3^{\mathrm{p}}$ points will be considered to establish a kriging response surface in the following. The optimization of the points distribution in the design space is achieved considering the Morris and Mitchell criterion [20] which maximizes the minimal distance between the sampling sites.

\section{B. Kriging model}

The advantage of the kriging modeling of a function, compared to other surrogate models lies in the estimation of the confidence in the interpolations made. It is affected by the distance between the unknown point $x$ and the sampling points and is expressed by the root mean square prediction error (RMSE). The response surface computed with kriging gives thus estimations of the function values at unsampled points but also a quantification of the confidence in these estimations. The kriging response surfaces modeling the variety of course corrections were constructed from the OpenMDAO toolbox which is an open-source mutli-objective optimization framework [21]. The enrichment of the model is achieved through the computation of the Expected improvement and the Probability of Feasibility described in the next paragraphs.

\section{Exploitation and exploration of the Kriging response surface}

To combine the local and global search of an optimum, Jones et al. [19] proposed the computation of the Expected Improvement $(E I)$ which is the mathematical definition of the probability that an unsampled point performs better than the already best known sampled point of the function. If the uncertainty at this unsampled point is modeled assuming a normal density function with mean $\hat{y}(x)$ and standard deviation $s(x)$ taken from the kriging model, the expression of EI is given by eq. (1) : 


$$
E[I(x)]=\left(y_{\min }-\hat{y}(x)\right) \Phi\left(\frac{y_{\min }-\hat{y}(x)}{s(x)}\right)+s(x) \phi\left(\frac{y_{\min }-\hat{y}(x)}{s(x)}\right)
$$

where $\Phi$ and $\phi$ are respectively the cumulative distribution and the standard normal density function. The EI proposes a trade-off between the exploration and the exploitation of the surrogate model. Indeed, values associated to the EI score are high in the regions of the design space where the function values are significant (exploitation) but are also high in the unknown regions of the model where the error associated to the model $s(x)$ is large (exploration).

\section{Constraints handling from the Kriging model}

The addition of constraint functions to the initial problem definition leads to the reduction of parameters combinations susceptible to minimize the objective function. They are modelled following the definition of the Probability of Feasibility (PF) introduced by Schonlau [22] and applied successfully to a surrogate-based optimization by Parr et al. [23]. In the same way as for the EI computation clarified in the preceding paragraph, the PF takes advantages of the kriging modeling of a function to estimate if constraints are likely to be respected by comparing the estimation of a function to the constraint limit, see eq. (2).

$$
P[F(x)]=\frac{1}{s(x) \sqrt{2 \pi}} \int_{0}^{\infty} e^{-\left[\left(G(x)-g_{\text {min }}\right)-\hat{g}(x)\right]^{2} /(2 \hat{s}(x))^{2}} d G
$$

where $g$ is the constraint function, $g_{\min }$ is the limit of the constraint and $G(x)-g_{\text {min }}$ is a measure of the feasibility. $G(x)$ is a random variable and $s(x)$ is constraint standard deviation of the Kriging model.

\section{Artificial Neural Networks}

A multi-layer Perceptron is specifically used in this study to model the evolution of the spoiler aerodynamic coefficients $\left(\Delta C_{A}, \Delta C_{N}\right.$ and $\left.\Delta C_{m}\right)$ as a function of the aerodynamic conditions (Mach number $M_{\infty}$ and angle of attack $\alpha$ ). The training database is composed of more than 1300 CFD evaluations of the spoiler. To avoid an over-fitting of the MLP, resulting in the degradation of the aerodynamic coefficients estimations, the training iterations stop when the coefficient of determination does no improve anymore on a subset of the training database (10\% of the evaluations in the present case).

The MLP models of each coefficient were implemented using the Python module Scikit-learn [24]. The details on the internal architecture providing the best reconstruction results (based on the calculation of the $R^{2}$ coefficient on a validation database) for the present study are gathered in Table 2 . It has to be noticed that the best estimations using MLP are obtained when the inputs and outputs are scaled in the same interval of range $[-1 ; 1]$. 


\begin{tabular}{|c|c|}
\hline MLP Layers & $5 \times 100 \times 100 \times 100 \times 3$ \\
\hline Optimization algorithm & LBFGS \\
\hline Activation function & Logistic sigmoïd $f(x)=\frac{1}{1+e^{-x}}$ \\
\hline
\end{tabular}

Table 2 MLP internal parameters

\section{Projectile aerodynamics}

\section{A. Simulations overview}

In this study, a MLP is used to model the variations in the aerodynamic coefficients of the spoiler configurations. The database is composed of RANS-based CFD evaluations. The contribution of the spoiler is isolated by simulating the flow around both controlled and uncontrolled projectile. The uncontrolled projectile has been studied prior to the present work, for instance by Simon et al. [25] and Zeidler et al. [26]. The aerodynamic modifications induced by the spoiler deployment are described in the following.

\section{Meshing strategy}

The $155 \mathrm{~mm}$ caliber (cal.) projectile is composed of a 5.6 cal-long body constituted of a 3 cal-long truncated nose. The 0.63 cal-long boat tail of the projectile is divided into a $3^{\circ}$ and a $7.5^{\circ}$ sloped part. 1300 RANS-based evaluations of the projectile aerodynamic coefficients are available for a wide variety of flight conditions and spoiler geometries to constitute an initial database.

The uncontrolled projectile computational grid is identical for every flow condition and is used as background mesh in the study of the controlled projectile. The computational domain external boundary extends at a distance of 50 caliber around the projectile. The total number of cells is equal to 22 million, the azimuthal resolution of the grid being set to 1.5 degree per cell. The spoiler setup on the projectile body is ensured via the chimera method which consists in merging two grids into a unique one as shown in fig. 3 . 


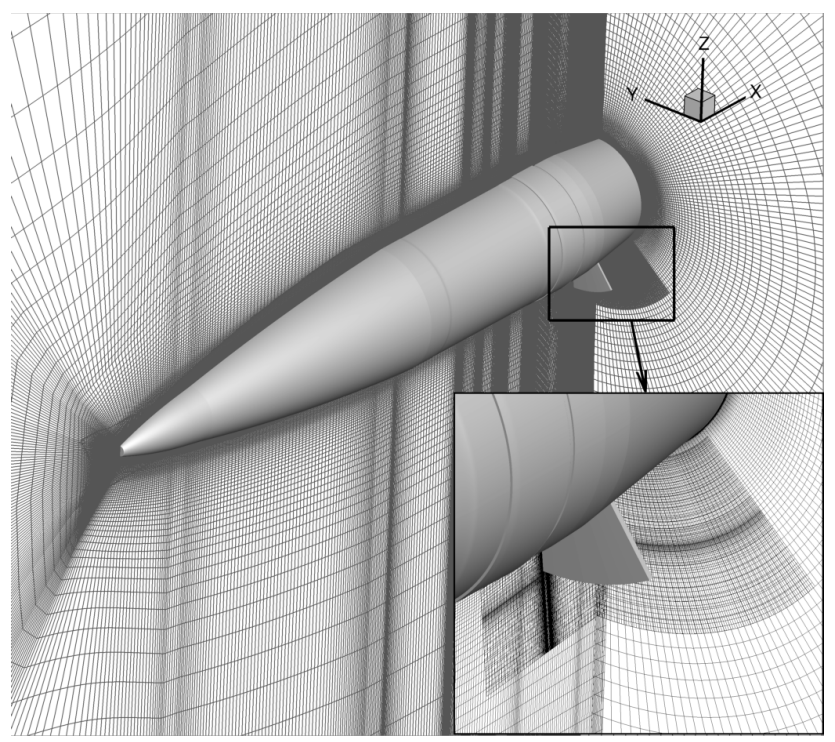

Fig. 3 Computational grid for the controlled projectile

Flow quantities are transferred from the background grid to the spoiler patch through interpolating areas. A cell-size ratio close to 1 at the interfaces between the background and the patch grids has been imposed. The grid associated to the spoiler is constituted of 5 million cells which leads to a controlled projectile mesh size of 27 million cells.

\section{2. elsA code}

The CFD solver used in this study to compute the aerodynamic coefficients of the different projectile configurations is the ONERA's solver elsA based on a cell-centered finite volume approach for solving the Navier-Stokes equations on structured multiblock grids [27]. RANS simulations are performed for a spoiler deployed under the projectile body to obtain positively defined pitching moment coefficient.

The computations constituting the initial aerodynamic coefficients database were realized using the Spalart-Allmaras (SA) one-equation turbulence model. Consistently the same turbulence model is used in the computations enhancing this database. It is known that SA model performs poorly in the presence of massively detached flows. However, a comparison with other turbulence models ( $k-\omega$ and EARSM not presented here) shows maximum discrepancies of the order of $10 \%$ for drag coefficient evaluations which is considered acceptable in the present case, since we are more interested in relative performance.

\section{B. Flow around the controlled projectile}

The flight conditions encountered by the uncontrolled projectile are ranging from supersonic Mach number (at the muzzle exit) to high subsonic, the angle of attack remaining below $6^{\circ}$. The deployment of the spoiler can occur at any point of the trajectory thus the uncontrolled and controlled projectile aerodynamic models are constructed in the same range of flight parameters. 
Four flight conditions were simulated corresponding to subsonic, transsonic and supersonic regimes. A generic spoiler is located in the middle of the projectile boat-tail to highlight the flow modifications induced by its presence. As shown in fig. 4 the topology of the flow remains similar to the uncontrolled projectile case with the presence of similar expansion and shock waves. The spoiler induces modifications in its vicinity and affects directly the projectile wake.

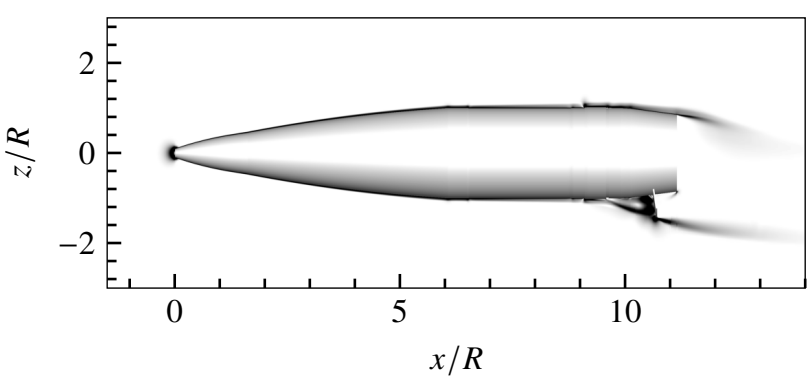

(a) $M=0.7$

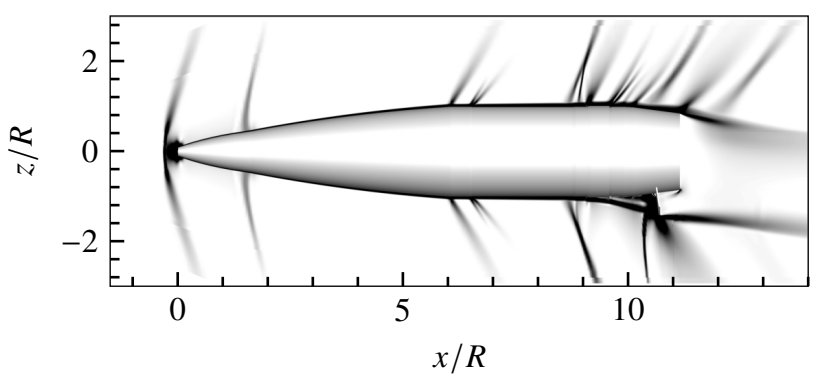

(c) $M=1.1$

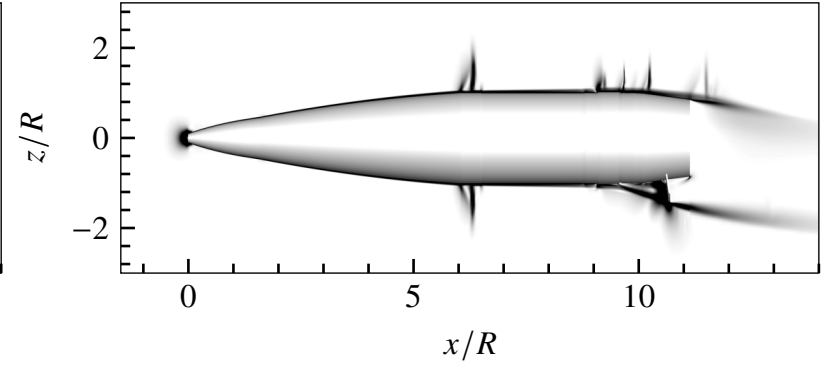

(b) $M=0.9$

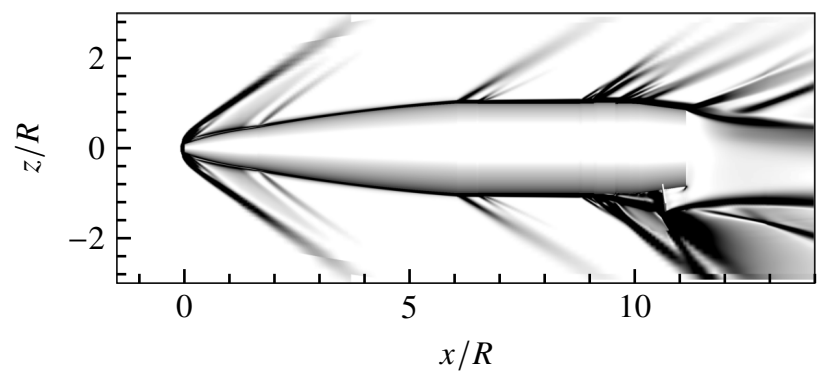

(d) $M=2.0$

Fig. 4 Controlled projectile, pseudo-schlieren visualization $\|\operatorname{grad}(\rho)\|$ at $\alpha=0^{\circ}$ for four freestream Mach numbers

Upstream of the spoiler a recirculation bubble is highlighted by the streamlines presented in fig. 5b. Downstream of the spoiler a second detached area is developed which merges with the wake of the projectile. Because of the longitudinal position of the spoiler close to the projectile base, a fluidic reattachment occurs in the computations of the present study. The diameter of the wake is increased compared to the uncontrolled projectile cases, resulting in an increase of the base drag. A comparison of streamlines is presented in fig. 5 focusing on the projectile base flow. The detached areas are showed for both projectile configurations at $M=0.9$ and $\alpha=0$. 


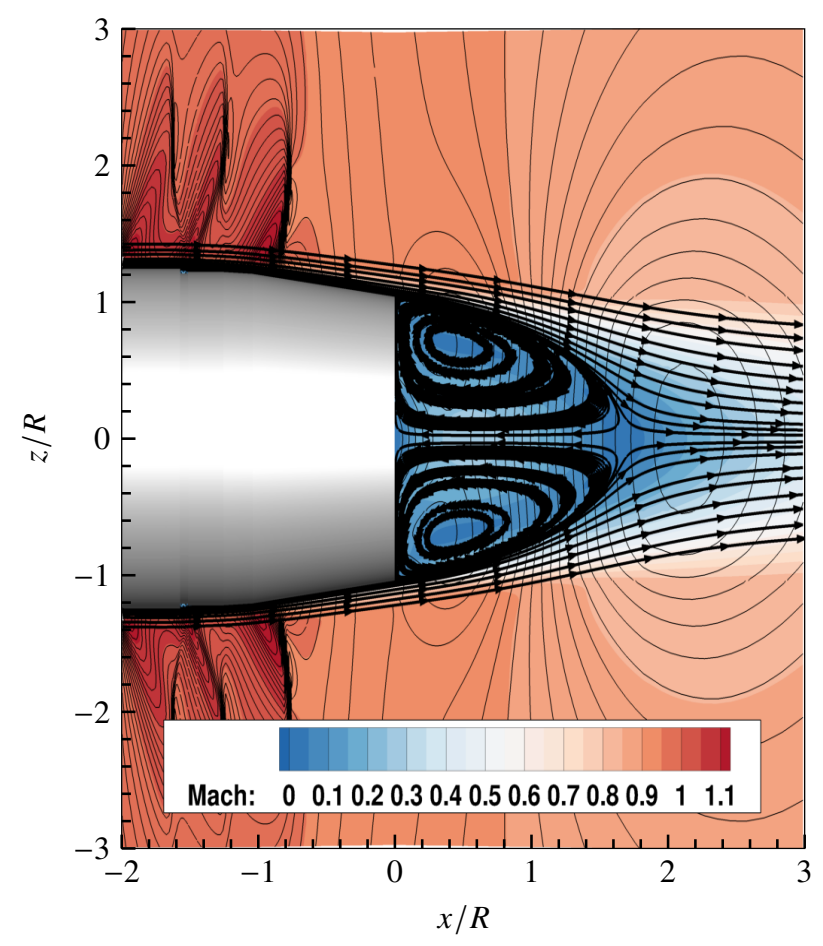

(a) Uncontrolled projectile base flow

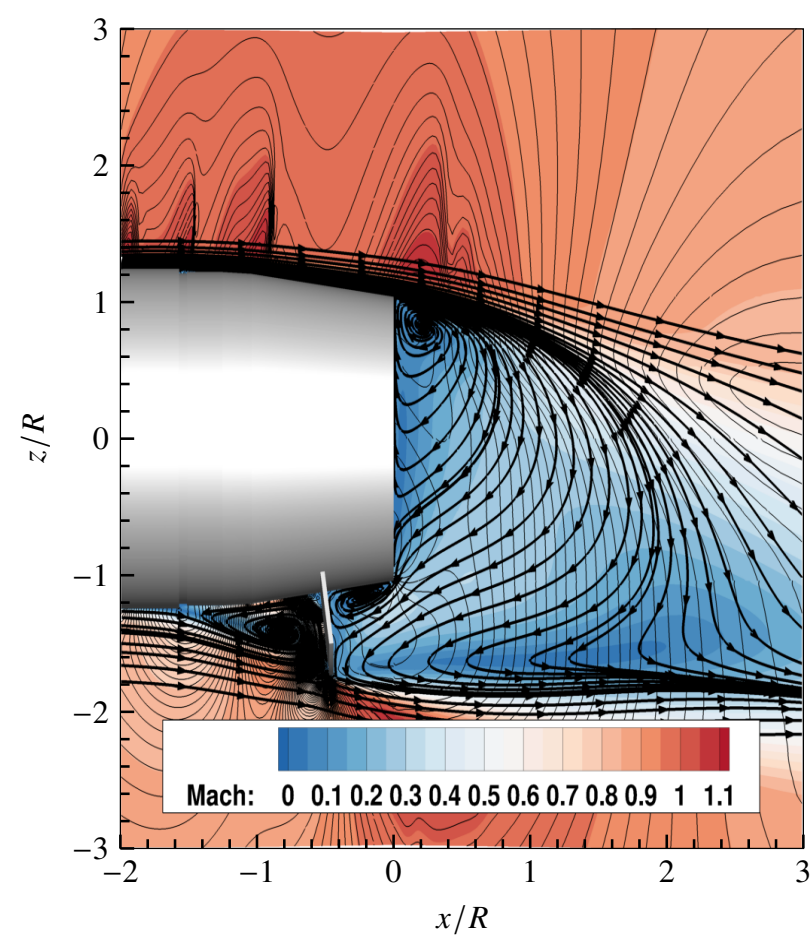

(b) Controlled projectile base flow

Fig. 5 Iso-contour of Mach number, iso-pressure lines and streamlines for $M=0.9$ and $\alpha=0^{\circ}$ in the symmetry plane

The symmetrical recirculation area defined by the streamlines downstream of the uncontrolled projectile base in fig. $5 \mathrm{a}$ is deeply modified by the presence of the spoiler as shown in fig. $5 \mathrm{~b}$. In the latter, the base flow is not symmetrical and the spoiler influence is perceivable in the intensity lowering and position modification of the shocks ahead of the boat-tail. Fig. $5 \mathrm{~b}$ highlights the modifications of the projectile trim angle with the disappearance of the stagnation point which is clearly identified in the case of the uncontrolled projectile 5a Directly downstream of the spoiler a small recirculation area is identified while on the other side, a recirculation bubble is formed just downstream of the base. Moreover, due to the curvature of the streamlines an additional shock appears in the wake which can be designated as recompression shock, for these transonic freestream conditions, which is not present in the case of the uncontrolled projectile.

\section{Spoiler optimization}

The optimization process developed within this study is illustrated by the flowchart presented in fig. 6 . In the specific optimization solved here, the instant of deployment of the spoiler is selected by the authors to happen in the last $12 \%$ of the uncontrolled projectile traveled distance. Within the $88 \%$ of the uncontrolled projectile course, information is gathered on the external perturbations occurring during the flight. The guidance system of the projectile computes the 
theoretical trajectory and thus determines accurately the expected impact point and the deviation from the uncontrolled projectile mean impact point.

The initial step of the process consists in estimating the spoiler contribution to the aerodynamic coefficients of the projectile with a MLP, paragraph $\mathrm{V} \mid \mathrm{A}$. Three response surfaces $\left(\Delta C_{A}, \Delta C_{N}\right.$ and $\left.\Delta C_{m}\right)$ are used in the trajectories computations by a six/seven degrees-of-freedom flight mechanics code of various configurations determined by LHS, paragraph $\mathrm{V} \mid \mathrm{B}$. These computations constitute the initialization step for the construction of kriging response surface of the trajectories modifications induced by the controlled surfaces, paragraph $\mathrm{V} / \mathrm{C}$. A sequential enrichment of the kriging response surfaces based on the selection of optimal design using a genetic algorithm is implemented to converge toward the optimum set of parameters achieving the required course correction, paragraph $\mathrm{V} \mid \mathrm{D}$. The main steps of this process are detailed in the following sections with illustrations of the ongoing optimization problem.

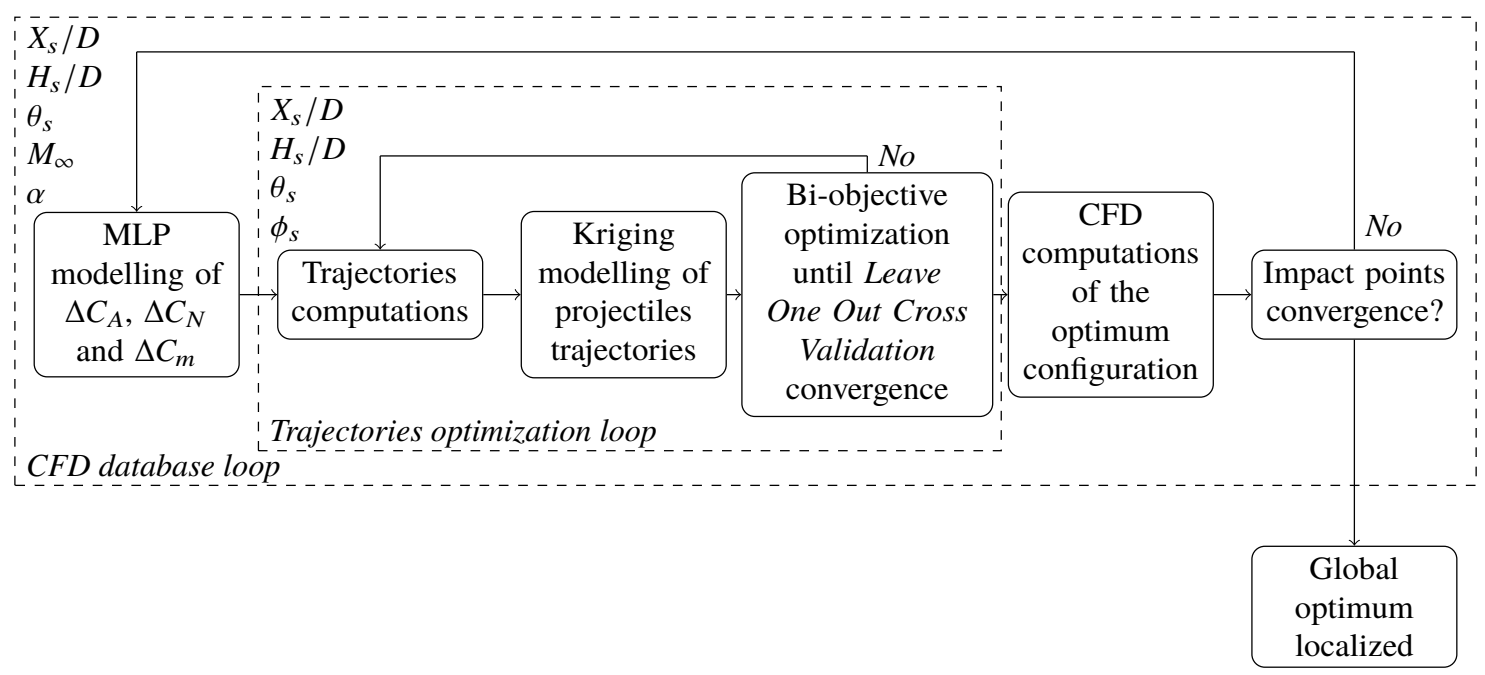

Fig. 6 Flowchart of the surrogate-based optimization algorithm

The trajectories optimization loop, referred to as inner loop in the following, defines which configuration of the controlled projectile is considered as optimum. During this process, the range and the lateral deviation produced by the selected configurations are added to the kriging databases used to model the objective and constraint functions. This inner loop constitutes the first adaptive sampling of the optimization process. The CFD database loop, referred to as outer loop, aims at refining the aerodynamic coefficients databases in the specific regions of the design space where promising configurations of the controlled projectile are identified. These databases are enriched during the global optimization process with the CFD based aerodynamic coefficients of the optimum configuration selected from the trajectories optimization loop until a state of convergence of the impact point position is reached. This enrichment is the second adaptive sampling. The MLP models associated to the aerodynamic coefficients of the control surface are trained with the additional CFD results before each new run of the inner loop. 


\section{A. Aerodynamic coefficients estimations}

A MLP is used to estimate the increment in force and moment due to the deployment of the spoiler $\left(\Delta C_{A}\right.$ for the additional axial force coefficient, $\Delta C_{N}$ for the additional normal force coefficient and $\Delta C_{m}$ for the additional pitching moment coefficient) over the 5 dimensions of the design space presented in table 3 ( 3 spoiler geometrical parameters $X_{s} / D, H_{s} / D, \theta_{s}$ to which the Mach number $M_{\infty}$ and the angle of attack $\alpha$ are added).

\begin{tabular}{|c|c|c|}
\hline \multirow{2}{*}{} & \multicolumn{2}{|c|}{ Parameter range } \\
\cline { 2 - 3 } & $\min$ & $\max$ \\
\hline Longitudinal position $X_{s} / D$ & 4.85 & 5.5 \\
\hline Height of the spoiler $H_{s} / D$ & 0.05 & 0.25 \\
\hline Span of the spoiler $\theta_{s}\left({ }^{\circ}\right)$ & 15 & 60 \\
\hline Mach number $M_{\infty}$ & 0.7 & 3 \\
\hline Angle of attack $\alpha\left(^{\circ}\right)$ & 0 & 6 \\
\hline
\end{tabular}

Table 3 MLP parameters range for the modeling of the spoiler aerodynamic coefficients

The initial MLP database consists of 1300 RANS computations. The advantage of using a MLP model against a kriging model here has been discussed by the authors in [28]. The construction of the kriging model required several hours while it is limited to twenty minutes when considering MLP. Additionally, the distribution of the CFD evaluations over the parameters space does not follow an optimal design of experiment which eventually leads to an over-fitting of the kriging model.

The validation of the MLP response surface training is illustrated in fig. 7 where MLP estimations of $\Delta C_{N}$ are compared to a validation set of 50 CFD evaluations, independent from the training database.
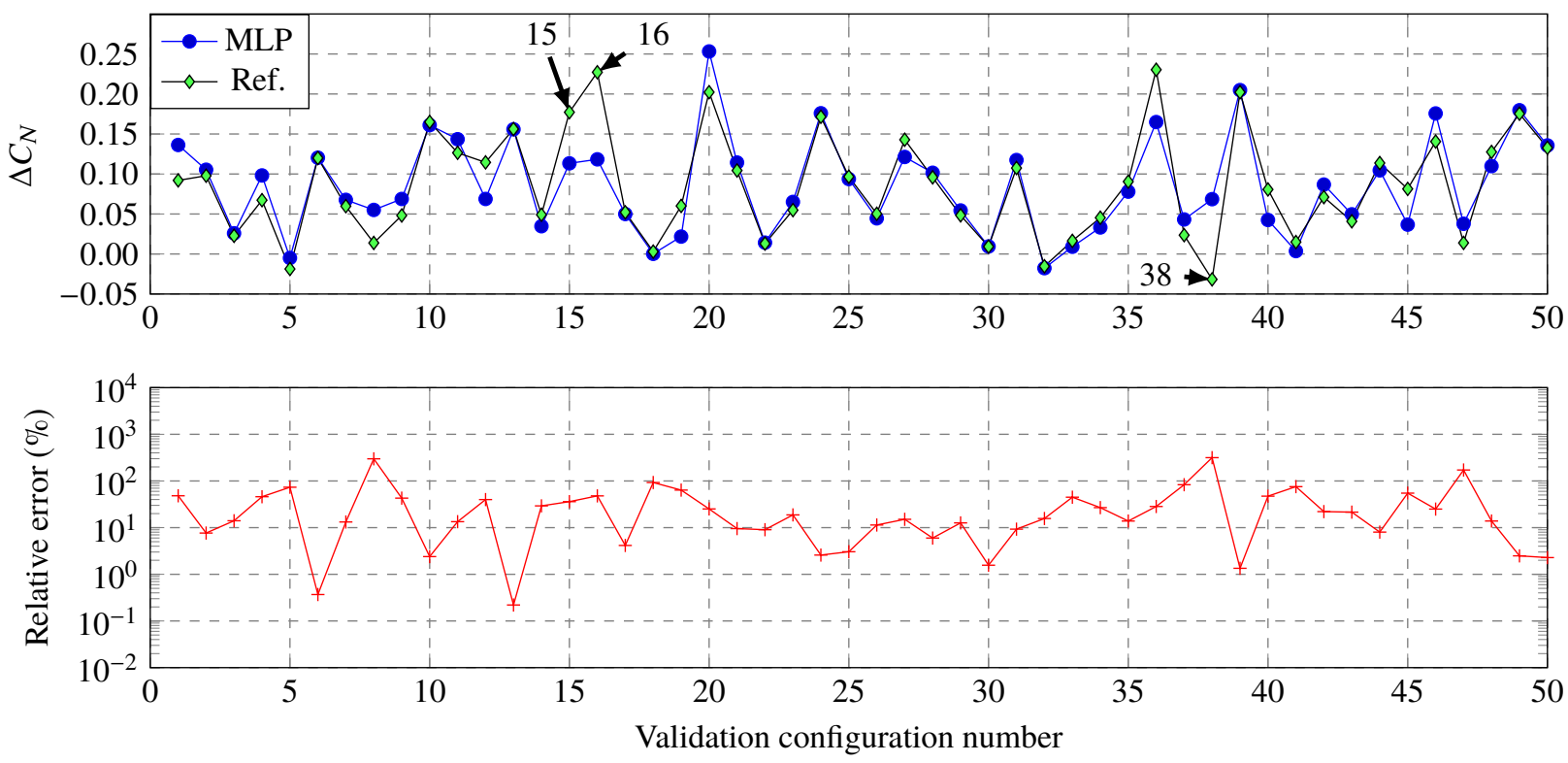

Fig. 7 MLP Lift force coefficient estimations over a validation database 
The quantification of the MLP quality is achieved by calculating the coefficient of determination $R^{2}$ which characterizes globally the surrogate model quality.

$$
R^{2}=1-\frac{\sum_{i=1}^{N}\left(\hat{y}\left(x_{i}\right)-y\left(x_{i}\right)\right)}{\sum_{i=1}^{N}\left(y\left(x_{i}\right)-\bar{y}(x)\right)}
$$

where $\hat{y}\left(x_{i}\right)$ is the estimation by the surrogate model at unsampled position $x_{i}, y\left(x_{i}\right)$ is the CFD value of the coefficient and $\bar{y}\left(x_{i}\right)$ is the mean over the $\mathrm{N}$ samples of the validation set. The initial MLP presents a $R^{2}=0.724$ for the lift coefficient however some estimations over the validation database present an important discrepancy compared to the actual value of the spoiler contribution. For instance, configurations 15, 16 or 38 show high value of the relative error. These particular spoiler geometries have one of their parameters very close to the boundaries defined in Table 1 which could explain the difficulty of the response surface to estimate accurately the coefficients.

The time needed to interpolate the aerodynamic coefficients using a MLP is only 30 seconds to get the 10164 combinations of $\alpha$ and $M_{\infty}$ required to compute a controlled projectile trajectory using the 6DoF code, which requires 20 seconds for each trajectory.

\section{B. Trajectories initial sampling}

An initial database of controlled projectiles is spread over the $p=4$ dimensional design domain ( 3 geometrical parameters $X_{S} / D, H_{S} / D, \theta_{s}$ and the roll position $\phi_{s}$ ) using the optimized LHS methodology. The number of samples is directly determined from the rule of thumb stated earlier as $N_{\text {init }}=3^{4}=81$ configurations. The trajectories are computed using the 6-DOF flight mechanics code Balco [29] and presented in fig. 8 .

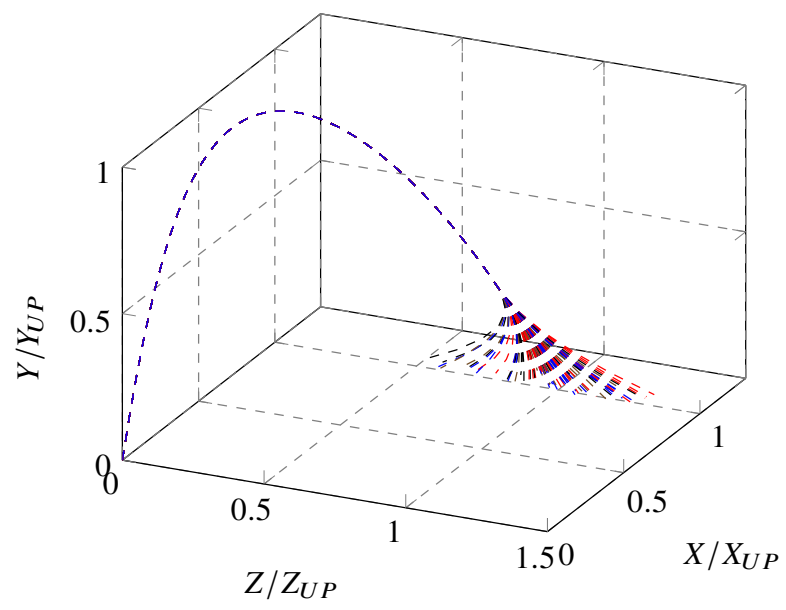

Fig. 8 Trajectories of controlled projectiles initialized by 4-dimensional LHS

A wide variety of corrections (around the uncontrolled projectile mean impact point at [1;1]) is proposed in this purely exploratory phase of the process. The lateral deviations and range modifications produced by the different spoilers are used to initialize the kriging response surfaces modeling the objective and constraint functions that are 
described in the next paragraph.

\section{Definition of the optimization problem}

Considering the distribution of the uncontrolled projectile impact points introduced in section $\Pi$, the objective function is defined as the minimization of the distance between the controlled projectile impact point and the closest point belonging to the $3 \sigma$ ellipse, eq. (4). Optimizing a spoiler geometry allowing such correction would ensure the correction of more than $99 \%$ perturbed trajectories. However, a single optimization of the objective function is not sufficient since any combination of range and lateral modification leading close to the $3 \sigma$ ellipse would be considered as optimum.

The optimization problem studied here consists in determining a spoiler geometry able to produce the course correction in the most unfavorable case of trajectory perturbation. It is then assumed that, if the spoiler geometry fulfills this specific case, it will allow producing every course correction, as illustrated in fig. 2 This is imposed through the definition of three additional constraints. An increase of the projectile range is imposed through eq. (5), illustrated in fig. 9 by the horizontal green dashed line. The natural motion of the projectile, directly influenced by its spinning, leads to a lateral deviation towards the right of the canon position. The selection of the most unfavorable case induces the reduction of this lateral deviation of the projectile, eq. [6, illustrated in fig. 9 by the vertical green dashed line.

Finally, to avoid an over-sizing of the spoiler geometry, only the configurations resulting in an impact point located inside the $3 \sigma$ ellipse are considered as optimum to the present problem. This constraint translates inot eq. (7) in which the distance between the controlled projectile impact point and the uncontrolled projectile mean impact point is compared to the distance between the uncontrolled projectile mean impact point and the closest point belonging to the $3 \sigma$ ellipse. It is illustrated in fig. 9 by the dashed green ellipse centered on the uncontrolled projectile mean impact point. 
Objective: $\min \left(f_{d}\right)=\sqrt{\left(X_{3 \sigma}-X_{C P}\right)^{2}+\left(Z_{3 \sigma}-Z_{C P}\right)^{2}}$

$$
\begin{array}{ll}
\text { w.r.t. constraints: } & X_{C P}>X_{U P}+2 \sigma_{X} \\
& Z_{C P}<Z_{U P}-2 \sigma_{Z} \\
& d\left(P_{U P} ; P_{C P}\right)<d\left(P_{U P} ; P_{3 \sigma}\right)
\end{array}
$$

\section{Definition of the objective and constraint functions}

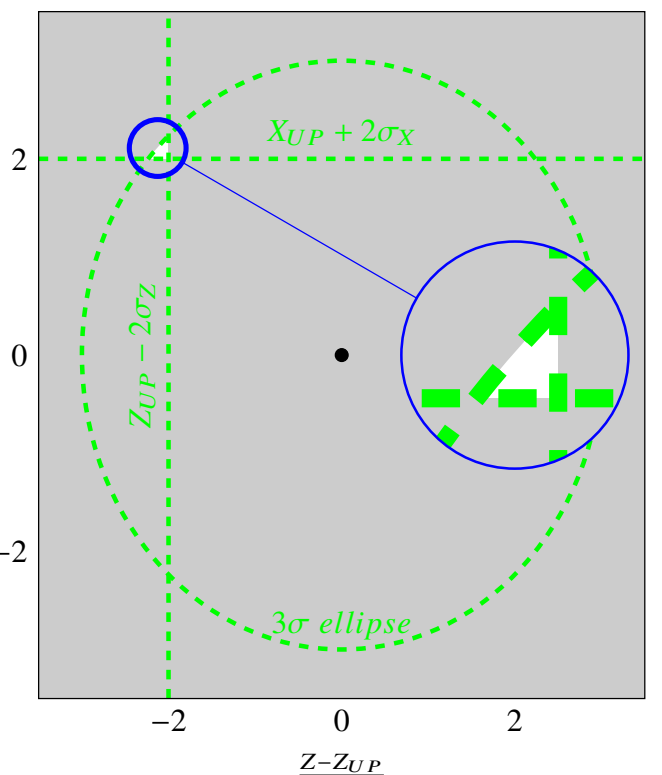

Fig. 9 Constrained targeted area for the course correction optimization problem

The grey area in fig 9 corresponds to a violation of a least one of the stipulated constraints. The targeted area is the tiny white circled zone where the optimizer should minimize the distance with the $3 \sigma$ ellipse. These colors for the constrained areas are used in the following presentation of the optimization results.

\section{Bi-objective optimization}

An adaptive sampling is developed inside the inner loop in fig. 6, based on the kriging estimations of the objective and constraint functions. A bi-objective optimization is achieved with the aim of maximizing $E I$ and $P F$ through an evolutionary process simulated with the NSGA-II algorithm developed by Deb et al. [30]. One specific configuration is selected at each step of the inner loop resulting from the convergence of the bi-objective genetic algorithm. Only individuals belonging to the Pareto front of this bi-objective optimization, illustrated for an on-going process in fig. 10 . can be selected to enrich the database. More specifically, following the suggestions by Parr et al. [31], the individual maximizing the product of both quantities is promoted, ensuring the exploration of the design space with configurations fulfilling the constraints. 


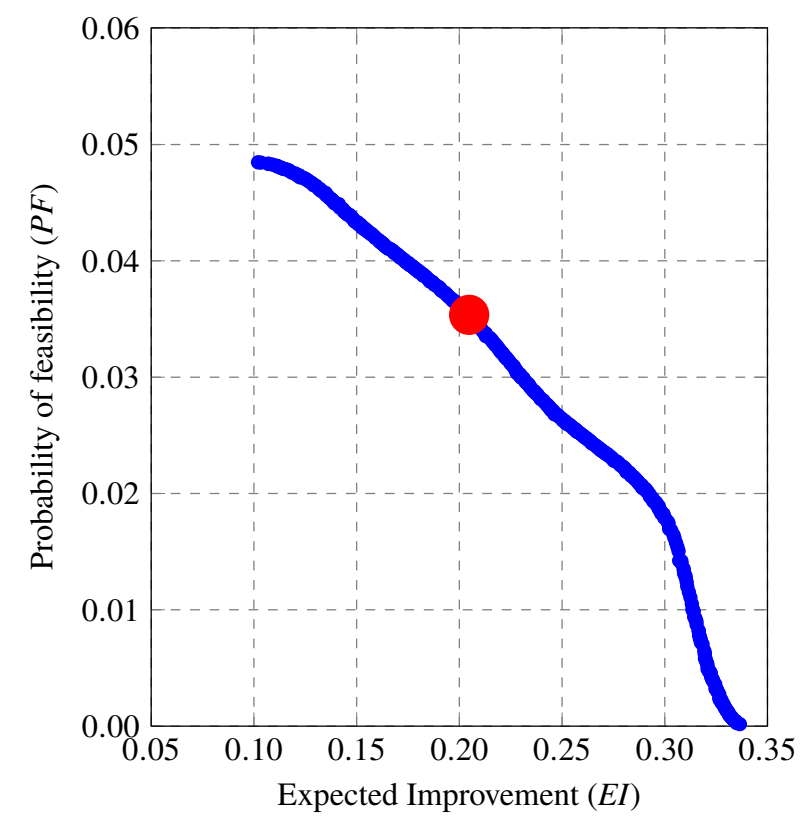

Fig. 10 Configurations of spoiler belonging to the Pareto Front during the inner loop optimization process, the red dot corresponds to the point of the front maximizing $E I \times P F$

The studies by Durantin [32] and Waldock [33] highlight the advantage of treating the problem as a multi-objective optimization. The maximization of EI and PF separately leads to the selection of better candidates than in the case of a single-objective optimization. Indeed, the product $E I \times P F$ is highly multi-modal, meaning that the optimizer may likely be trapped in the neighborhood of a local optimum while this behavior can be avoided in the multi-objective optimization. Thus, it is important to note that the point selected from the single-objective optimization may not correspond to the optimum found on the Pareto Front of the multi-objective optimization.

\section{E. Course correction results}

The adaptive sampling described in the previous section is applied to the projectile course correction problem. A convergence criterion, chosen as the evaluation of the Leave One Out Cross Validation (Myers et al. [34]), is compared to a threshold $\left(10^{-4}\right.$ in the present study) for the kriging modeling of the objective function. The adaptive sampling of the optimization is conducted until this criterion is reached. The distance of the impact point to the $3 \sigma$ ellipse for all the configurations satisfying the constraints is evaluated. The configuration leading to the closest point to the $3 \sigma$ ellipse is selected as an 'intermediate' optimum and its aerodynamic coefficients $\left(\Delta C_{A}, \Delta C_{N}\right.$ and $\left.\Delta C_{m}\right)$ are assessed using RANS computations.

The spreading of the impact points in the targeted area is presented in fig. 11 from the initialization step to the last iteration of the process which occurs after 3 CFD enrichment of the MLP databases. The impact points are colored by the spoiler frontal surface associated to the trajectory modification. 
Frontal surface $\left(\mathrm{m}^{2}\right)$
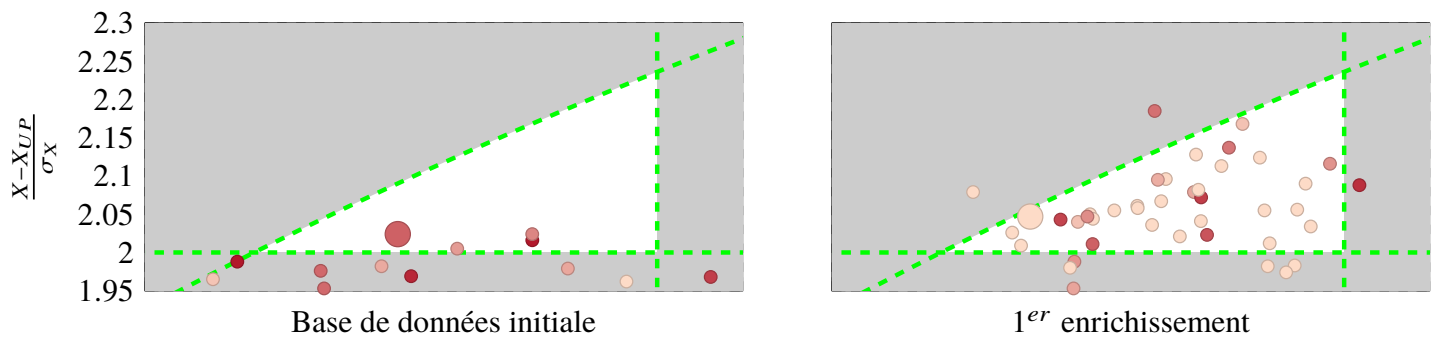

0.0036

0.0032

0.0028

0.0024

0.0020
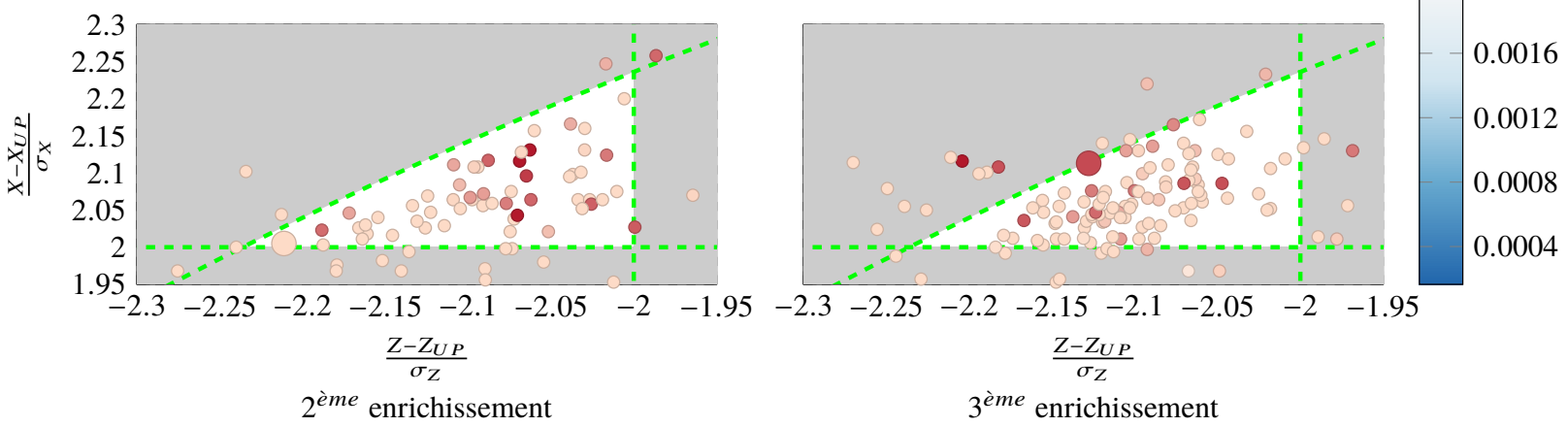

Fig. 11 Resulting impact points during the optimization process, dots are colored by the spoiler frontal surface of the spoiler

The initial stage of the optimization is only able to select 4 configurations of the spoiler producing the required range increase $\frac{X-X_{U P}}{2 \sigma_{X}}$ eq. 5 before the convergence threshold on the objective response surface is reached. The configurations of the spoiler presented in fig. 11, after the enrichment of the MLP database, are almost equivalent regarding the spoiler frontal surface. This means that in the 4-dimensional design space, the configurations producing the required 2-D course correction are located on an hypersurface depending on $H_{S} / D \times \theta_{s}$. The choice of any spoiler configuration belonging to this particular hypersurface determines the magnitude of the force induced by the spoiler. The longitudinal position of the spoiler $X_{S} / D$ influences the magnitude of the pitching moment created by the spoiler which is directly linked to the lateral deviation produced by a spinning projectile while the roll position $\phi_{s}$ determines toward which direction the force induced by the spoiler is applied.

A refinement of the MLP database occurs between two steps of the optimization process. Contributions of the spoiler to the aerodynamic coefficients is deduced from CFD computations spread along the controlled part of the trajectory following a LHS. An example of this second adaptive sampling is illustrated in fig. 12 where the seven red dots represent the flow conditions for the CFD computations of the aerodynamic coefficients. Initially, nine sampling point were selected for the adaptive refinement of the MLP models. However, it appeared that similar flight conditions were selected for three CFD computations leading to almost identical spoiler contribution to the aerodynamic coefficients. Seven aerodynamic conditions were chosen to enrich the MLP database, thus avoiding an eventual over-fitting of the surrogate model. 


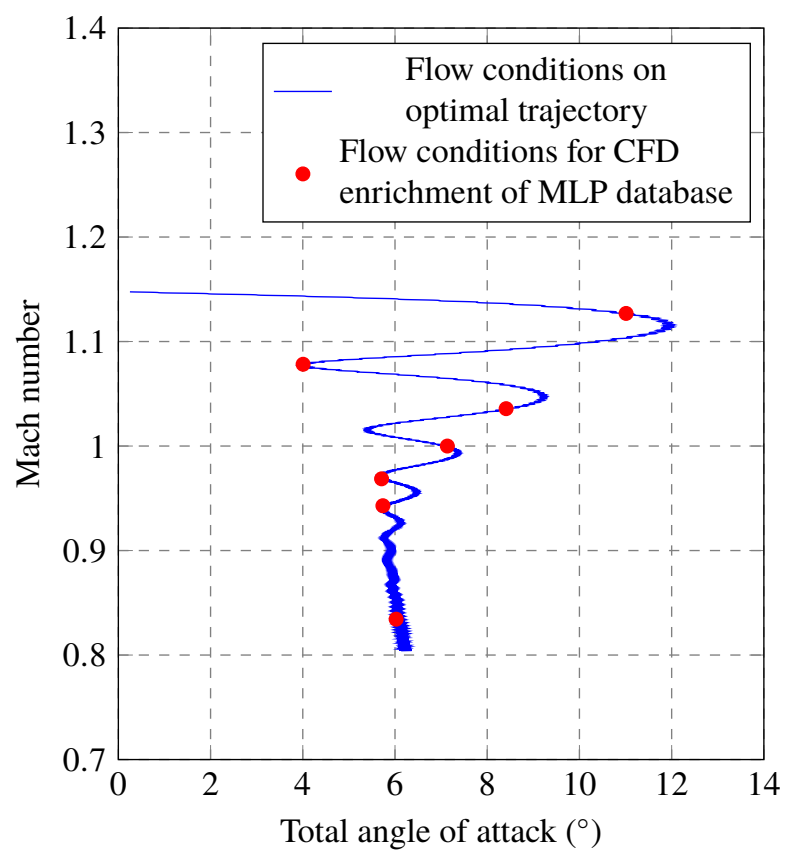

Fig. 12 Choice of flow conditions during the controlled projectile flight identified on the optimal trajectory defined by the inner loop

In the present case, a convergence state on the impact point position is reached after 3 CFD enrichment of the MLP databases. The use of surrogate models allows solving an inner loop step in 24 hours. Each of the CFD enrichment requires between 30 and 45 hours over 84 processor for each flow conditions $(45 \times 84 \times 7=26460 \mathrm{CPU}$ hours for one step of CFD enrichment). The global optimum configuration is capable of producing a course correction very close to the $3 \sigma$ ellipse while satisfying the constraints, hence able to correct most of the trajectories.

The enrichment of the MLP databases between two inner loop steps, illustrated in fig. 11, has a clear influence on the number of identified controlled projectiles able to produce the required 2-D course correction and results in impact points getting closer to the $3 \sigma$ ellipse. Indeed, refining the MLP database by computing the optimum configuration aerodynamic coefficient, which hence belongs to the hypersurface of equal frontal surface, leads to an increase in the estimations accuracy for all the configurations belonging to the subset of potential solutions and results finally in the identification of an optimum spoiler configuration.

\section{Flight Mechanics study of the optimum configuration}

The optimum configuration determined during the process enables the course correction of the projectile. The constraints defined in the problem statement conflict with the natural motion of the uncontrolled projectile. The modifications of the projectile behavior allowing an increase in the range and a decrease in the lateral deviation after the deployment of the spoiler are highlighted in this section.

The flight mechanics behavior of the controlled projectile was unknown prior to the present study. This explains the 
restriction of the initial MLP database angle of attack definition to a maximum of $6^{\circ}$. Indeed, at the maximum the uncontrolled projectile angle of attack is $\alpha=3^{\circ}$ as illustrated in fig. 13a. As a consequence, an increase was expected once the spoiler is deployed but it appears in fig. $13 \mathrm{a}$ that it exceeds $10^{\circ}$ during the trajectory. The deployment of the spoiler during the flight leads to an extrapolation of the aerodynamic coefficients by the initial MLP response surfaces. The interest of the optimization outer loop is thus, in a first time, to extend the domain of definition of the MLP database. Moreover, the choice of a specific spoiler instant of deployment (12\% of the trajectory before the impact in the studied case) in combination with a specific frontal surface value leads to the identification of designs able to produce the required 2-D course correction. In the 4 dimensional domain (defined in table 1), an iso-surface of spoiler frontal surface is thus defined by the product $F S_{s}=\theta_{s} \times H_{s} / D$. In the present course correction problem, this value is lower than the maximum allowed by the parameters range, as indicated by the parameters combination in table $14 \mathrm{a}$ The adjustment of the instant of deployment for a given spoiler geometry with the modification of the roll angle $\phi_{s}$ allows producing any 2-D course correction. The magnitude of the trajectory modifications depends thus on the integration of the additional forces induced by the spoiler (which are maximized when the frontal surface is maximum) over the time interval during which it is deployed.

After the computation of the first inner loop, the extension of the MLP database is thus beneficial for all the evaluated configurations. The following iterations of the outer loop are used to increase the accuracy of the MLP database until a convergence state is reached. The modification of the aerodynamic coefficient induced by the enrichment of the database is shown in fig. 13 where the evolution of the optimum projectile coefficients are presented. It has to be noted that in fig. $13 \mathrm{~b}$ and $13 \mathrm{c}$ the aerodynamic coefficients of the uncontrolled projectile are close to 0 while the figures focuses on the spoiler contributions. 

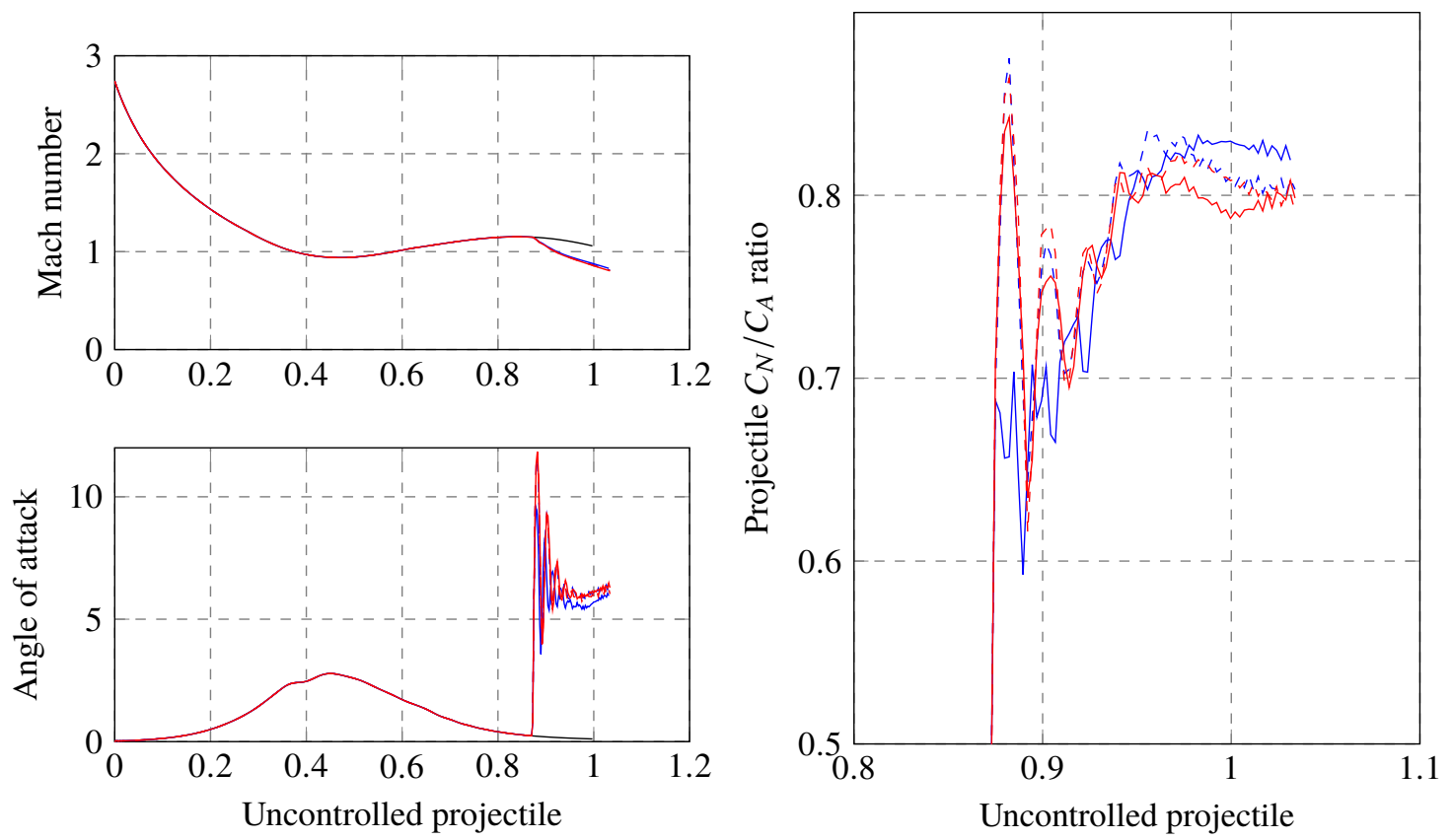

Non-dimensionalized flight time

Non-dimensionalized flight time

(a) Aerodynamic conditions

(b) $C_{N} / C_{A}$

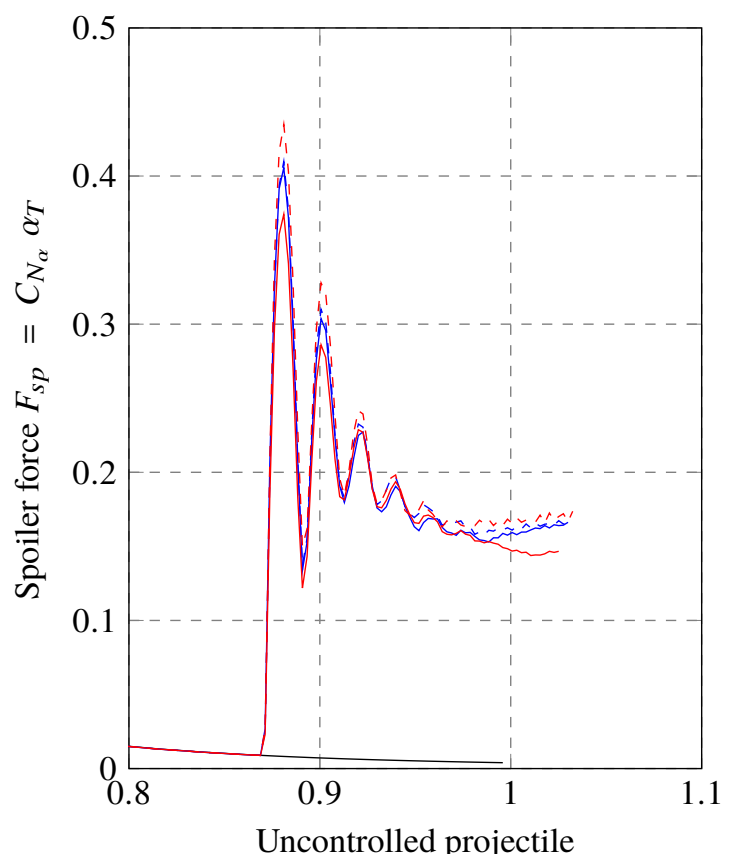

Uncontrolled projectile

Non-dimensionalized flight time

(c) $C_{N_{\alpha}} \alpha_{T}$

Fig. 13 Evolution of the aerodynamic coefficients of the optimum controlled configuration through the enrichment of the MLP database, uncontrolled projectile $(-)$, initial database $\left(-\frac{-}{-}, 1^{\text {st }}\right.$ iteration (- - - ), $2^{\text {nd }}$ iteration (- $), 3^{\text {rd }}$ iteration (- - - ) 
The formulation of the optimization problem constraints requires an extension of the projectile range. The deployment of a spoiler leads naturally to an increase in the drag coefficient $C_{A}$ of the controlled projectile, which reduces the range if the device roll position is not piloted. One solution to achieve the range extension goal is thus to increase the lift to drag ratio of the projectile $C_{N} / C_{A}$ as illustrated in fig $13 \mathrm{~b}$. This is achieved by the increase angle of attack highlighted in fig. 13a An additional force $F_{s} p$ is induced by the spoiler deployment, which is diametrically opposed to its roll position. The trajectory modification results in the integration of this force over the time interval during which the spoiler is deployed, as expressed in eq. (8) :

$$
\begin{aligned}
z & =\int_{t_{\text {depl }}}^{t_{\text {imp }}} F_{\text {sp }} d t=\int_{t_{\text {depl }}}^{t_{\text {imp }}} \frac{1}{2} \rho S V^{2} C_{N \alpha} \alpha_{T} d t \\
\text { with } \alpha_{T} & =\sqrt{\alpha^{2}+\beta^{2}}
\end{aligned}
$$

with $\alpha_{T}$ (the total angle of attack) the root-square-sum of the pitch and yaw angles. The ability of controlling the spoiler roll position $\phi_{s}$ enables orienting this additional force and thus the course correction.

The optimum configuration obtained from the algorithm combines the two behaviors described above to achieve the required 2-D course correction. The geometrical characteristics of the optimum are gathered in Table $14 \mathrm{a}$ and presented in fig. $14 \mathrm{~b}$ :

\begin{tabular}{|c|c|}
\hline & Parameter value \\
\hline Longitudinal position $X_{S} / D$ & 5.494 \\
\hline Height of the spoiler $H_{s} / D$ & 0.247 \\
\hline Span angle $\theta_{s}\left({ }^{\circ}\right)$ & 58.3 \\
\hline Roll position $\phi_{s}\left(^{\circ}\right)$ & 352.5 \\
\hline
\end{tabular}

(a) Definition of the optimum spoiler geometry

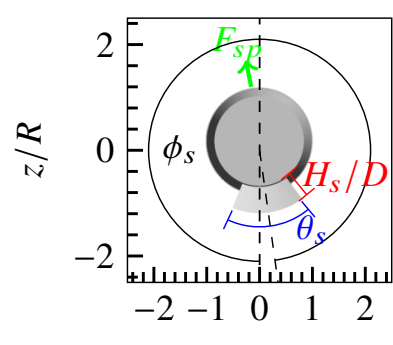

$x / R$

(b) Optimum spoiler geometry parameters

\section{Fig. 14 Resulting spoiler producing a 2-D course correction}

The deployment of the spoiler induces the production of an aerodynamic force directly opposed to its position on the body of the projectile, represented by the vector $F_{s p}$ in fig. $14 \mathrm{~b}$ b. This additional force can be decomposed into a lift and a lateral force contribution. Due to the constraints imposed in the present optimization process, the spoiler is deployed under the projectile body. The normal forces induced by the spoiler and the projectile are thus oriented in the same direction, maximizing the controlled projectile lift. These contributions to the aerodynamic forces acting on the projectile result respectively in an increase in range and to a reduction of the lateral deviation to produce the required 2-D course correction. 


\section{Conclusions}

A surrogate-based algorithm was developed with the aim of optimizing a spoiler geometry deployed during the flight of a spin-stabilized projectile. An original feature of the present study consists in the coupling of MLP and kriging response surfaces with a flight mechanics code to identify spoiler geometries able to produce a specified course correction. The selection of an optimum configuration is achieved through several adaptive sampling of the different response surfaces. Kriging response surfaces are enriched in the inner loop of the optimization problem to model the objective and constraint functions while the MLP database is enhanced with additional CFD evaluations of the optimum spoiler aerodynamic coefficients. The controlled projectile is able to reach an increased range and a reduced lateral deviation compared to the uncontrolled projectile trajectory. The modification of the angle of attack consecutive to the spoiler deployment leads to the increase of the coefficients ratio responsible for the trajectory modifications.

Even if it would have been more rigorous to initially set a wider angle range of angle of attack for the MLP database, the outer loop is able to circumvent this issue. The methodology adapted to the precision error reduction is flexible since an adaptation of the objective function modeled by kriging allows solving an optimization problem for any course correction type. This method could be complemented with a stability analysis of the controlled projectile flight.

In the following of this study, multi-fidelity surrogate models could be used to integrate unsteady computations of the optimum configuration in the databases. Kriging variants, such as Hierarchical kriging or Co-kriging could be used to estimate the contribution of the spoiler to the aerodynamic coefficients with increased accuracy. Moreover, wind tunnel measurements of the controlled projectile aerodynamic coefficients will be compared with the surrogate models estimations.

\section{Acknowledgment}

This study was partly funded by Nexter Munitions and by ONERA, The French Aerospace Lab. It contributes to the DGA funded research project "Manege II" which is a collaborative work between ONERA, Nexter Munitions and Institut Saint Louis. The authors would like to particularly acknowledge R. Thepot and P. Denis and all the contributors to the Manege II project, specifically E. Carette for all the discussions about projectile we had during this study.

\section{References}

[1] Dietrich, F., "Simulation numerique du couplage entre la mecanique du vol et l'aerodynamique des projectiles," Ph.D. thesis, Universite de Poitiers, 2003.

[2] Whyte, H. R., “SPIN-73 an Updated Version of the SPINNER Computer Program,” Tech. rep., Picatinny Arsenal, 111973.

[3] Paiva, R., Crawford, C., and Suleman, A., "Comparison of Surrogate Models in a Multidisciplinary Optimization Framework for Wing Design,” AIAA Journal, Vol. 48, 2010, pp. 995-1006. doi:10.2514/1.45790. 
[4] Peter, J., Marcelet, M., Burguburu, S., and Pediroda, V., "Comparison of surrogate models for the actual global optimization of a 2D turbomachinery flow," Proceedings of the 7th WSEAS International Conference on Simulation, Modelling and Optimization, 2007 , pp. $46-51$.

[5] Rajkumar, T., and Bardina, J., "Prediction of Aerodynamic Coefficients Using Neural Networks for Sparse Data," FLAIRS 2002 Proceedings, 2002, pp. 242-246.

[6] Meunier, M., "Simulation and Optimization of Flow Control Strategies for Novel High-Lift Configurations," AIAA Journal, Vol. 47, No. 5, 2009, pp. 1145-1157. doi:10.2514/1.38245.

[7] Kanazaki, M., Tanaka, K., Jeong, S., and Yamamoto, K., "Multi-Objective Aerodynamic Exploration of Elements' Setting for High-Lift Airfoil Using Kriging Model,” Journal of Aircraft, Vol. 44, No. 3, 2007, pp. 858-864. doi:10.2514/1.25422.

[8] Jeong, S., Murayama, M., and Yamamoto, K., "Efficient Optimization Design Method Using Kriging Model," Journal of Aircraft, Vol. 42, No. 2, 2005, pp. 413-420. doi:10.2514/1.6386.

[9] Forrester, A. I. J., Bressloff, N. W., and Keane, A. J., “Optimization using surrogate models and partially converged computational fluid dynamics simulations," Proceedings of the Royal Society A, Vol. 462, edited by P. R. S. A, 2006, pp. 2177 - 2204. doi:10.1098/rspa.2006.1679.

[10] Schönning, M. A., Nayfeh, J. F., and Zarda, P. R., "Using Response Surface Approximations to Cover Holes in the Design Space: Conceptual Design of a Missile," Journal of Computing and Information Science in Engineering, Vol. 2, No. 3, 2003, pp. 224-231. doi:10.1115/1.1527056.

[11] Won, H., Pfaender, H., Levine, S., and Mavris, D., "Using Response Surface Equations to Optimize the Aerodynamics of a High Speed Standoff Missile Within a Multi-Disciplinary Analysis Setting," AIAA Aircraft Technology, Integration, and Operations (ATIO), 2002.

[12] Fowler, L., and Rogers, J., “Airframe Performance Optimization of Guided Projectiles Using Design of Experiments,” Journal of Spacecraft and Rockets, Vol. 52, No. 6, 2015, pp. 1603-1613. doi:10.2514/1.A33367.

[13] Patel, M., DiCocco, J., Prince, T., and Ng, T., “Afterbody Flow Control for Low-Alpha Missile Maneuvering,” 21st AIAA Applied Aerodynamics Conference, 2003. doi:10.2514/6.2003-3673.

[14] Simon, F., "Simulations numériques hybrides RANS/LES de l'aérodynamique des projectiles et application au contrôle des écoulements," Ph.D. thesis, Université des sciences et technologies de Lille 1, 2007.

[15] “The NATO Armaments Error Budget," Tech. rep., August 2011.

[16] Wey, P., Martinez, B., Cayzac, R., Carette, E., Denis, P., and Grignon, C., “2D Course Correction System for Spin-Stabilized Projectiles Using a Spoiler Control Surface,” 28th International Symposium on Ballistics, 2014. 
[17] Swiler, L., Slepoy, R., and Giunta, A., "Evaluation of Sampling Methods in Constructing Response Surface Approximations," Structures, Structural Dynamics, and Materials and Co-located Conferences, American Institute of Aeronautics and Astronautics, 2006. doi:10.2514/6.2006-1827.

[18] Mckay, D. M., Beckman, R., and Conover, W., "A Comparison of Three Methods for Selecting Values of Input Variables in the Analysis of Output From a Computer Code," Technometrics, Vol. 21, 1979, pp. 239-245. doi:10.1080/00401706.1979.10489755.

[19] Jones, D. R., Schonlau, M., and Welch, W. J., "Efficient Global Optimization of Expensive Black-Box Functions,” Journal of Global Optimization, Vol. 13, No. 4, 1998, pp. 455-492. doi:10.1023/A:1008306431147.

[20] Morris, M. D., and Mitchell, T. J., "Exploratory designs for computational experiments,” Journal of Statistical Planning and Inference, Vol. 43, No. 3, 1995, pp. 381 - 402. doi:https://doi.org/10.1016/0378-3758(94)00035-T.

[21] Gray, J. S., Moore, K. T., and Naylor, B. A., “OpenMDAO: An Open Source Framework for Multidisciplinary Analysis and Optimization," Proceedings of the 13th AIAA/ISSMO Multidisciplinary Analysis Optimization Conference, 2010. AIAA 2010-9101.

[22] Schonlau, M., “Computer Experiments and Global Optimization,” Ph.D. thesis, University of Waterloo, 1997.

[23] Parr, J. M., Keane, A. J., Forrester, A. I., and Holden, C. M., "Infill sampling criteria for surrogate-based optimization with constraint handling," Engineering Optimization, Vol. 44, No. 10, 2012, pp. 1147-1166. doi:10.1080/0305215X.2011.637556.

[24] Pedregosa, F., Varoquaux, G., Gramfort, A., Michel, V., Thirion, B., Grisel, O., Blondel, M., Prettenhofer, P., Weiss, R., Dubourg, V., Vanderplas, J., Passos, A., Cournapeau, D., Brucher, M., Perrot, M., and Duchesnay, E., “Scikit-learn: Machine Learning in Python,” Journal of Machine Learning Research, Vol. 12, 2011, pp. 2825-2830.

[25] Simon, F., Deck, S., Guillen, P., Cayzac, R., and Merlen, A., "Zonal-Detached-Eddy Simulation of Projectiles in the Subsonic and Transonic Regimes,” AIAA Journal, Vol. 45, No. 7, 2007, pp. 1606-1619. doi:10.2514/1.26827.

[26] Zeidler, M., Garnier, E., Cayzac, R., and Merlen, A., "Fluidic Control of a 155 Millimeter Spin-Stabilized Projectile Using Coanda Effect,” AIAA Journal, Vol. 53, No. 5, 2015, pp. 1146-1158. doi:10.2514/1.J053085.

[27] Cambier, Laurent, Heib, Sébastien, and Plot, Sylvie, "The Onera elsA CFD software: input from research and feedback from industry," Mechanics \& Industry, Vol. 14, No. 3, 2013, pp. 159-174. doi:10.1051/meca/2013056.

[28] Arnoult, G., Zeidler, M., and Garnier, E., “Optimization Methodology for a 2-D Course Correction of a 155 mm Spin-Stabilized Projectile," AIAA AVIATION Forum, American Institute of Aeronautics and Astronautics, 2018. doi:10.2514/6.2018-3950.

[29] Wey, P., Corriveau, D., Saitz, T. A., de Ruijter, W., and Strömbäck, P., "BALCO 6/7-DoF TRAJECTORY MODEL,” 29th International Symposium on Ballistics, Vol. 1, 2016, pp. 151 - 162.

[30] Deb, K., Pratap, A., Agarwal, S., and Meyarivan, T., "A fast and elitist multiobjective genetic algorithm: NSGA-II,” IEEE Transactions on Evolutionary Computation, Vol. 6, No. 2, 2002, pp. 182-197. doi:10.1109/4235.996017. 
[31] Parr, J. M., Forrester, A. I. J., Keane, A. J., and Holden, C. M. E., "Enhancing Infill Sampling Criteria for Surrogate-based Constrained Optimization," J. Comp. Methods in Sci. and Eng., Vol. 12, No. 1-2, 2012, pp. 25-45. doi:10.3233/JCM-2012-0402.

[32] Durantin, C., Marzat, J., and Balesdent, M., "Analysis of multi-objective Kriging-based methods for constrained global optimization," Computational Optimization and Applications, Vol. 63, No. 3, 2016, pp. 903-926. doi:10.1007/s10589-0159789-6.

[33] Waldock, A., and Corne, D., "Multiple Objective Optimisation Applied to Route Planning," Proceedings of the 13th Annual Conference on Genetic and Evolutionary Computation, ACM, New York, NY, USA, 2011, pp. 1827-1834. doi: $10.1145 / 2001576.2001821$.

[34] Myers, R., Montgomery, D., and Anderson-Cook, C., Response Surface Methodology: Process and Product Optimization Using Designed Experiments, Wiley Series in Probability and Statistics, Wiley, 1995. 